\title{
QUANTITATIVE STUDY OF SYNAPTIC BOUTONS ON FROG INTRACARDIAC NEURONS
}

\author{
Darius Batulevičius, Gertrūda Skripkienė, \\ Denas Andrijauskis, Berta Kėryté, Valdas Skripka \\ Institute of Anatomy, Faculty of Medicine, \\ Lithuanian University of Health Sciences, Kaunas, Lithuania
}

\begin{abstract}
The frog is a useful model to study the structure and function of intracardiac neurons. The goal of this study was to evaluate the size and distribution of synaptic boutons on the intracardiac neurons in the frog Rana temporaria. Interatrial septa from four animals were double-labelled immunohistochemically for the cholinergic marker choline acetyltransferase (ChAT) and the marker of synaptic vesicles synaptophysin (SYP). One hundred intracardiac neurons were analysed by confocal microscopy. Terminals of preganglionic axons were strongly positive for ChAT, while synaptic boutons were strongly positive for both ChAT and SYP. The number of synaptic boutons per neuron ranged from 2 to 121 and was $10 \pm 2$ (mean \pm SE). The total area of synaptic boutons ranged from $6 \mu \mathrm{m}^{2}$ to $270 \mu \mathrm{m}^{2}$ and was $98 \pm 6 \mu \mathrm{m}^{2}$. The largest total area of synaptic boutons was found on the axonal half of neuronal soma $\left(59 \pm 4 \mu \mathrm{m}^{2}\right)$. The total areas of synaptic boutons on both the non-axonal half of soma and the proximal axon were smaller $\left(36 \pm 3 \mu \mathrm{m}^{2} ; 6 \pm 2 \mu \mathrm{m}^{2} ; \mathrm{P}<0.001\right)$. Synaptic boutons occupied $13 \pm 1 \%$ of the area of the neuronal soma profile. Conclusions: 1) The axonal half of the soma of the frog intracardiac neuron is more densely innervated than the non-axonal half of the soma. 2) The axosomatic and axoaxonic synapses are present on frog intracardiac neurons. The study provides a framework for further experimental studies on the formation and rearrangement of synapses on frog intracardiac neurons.
\end{abstract}

Keywords: heart; autonomic; ganglion; cholinergic; synapse; axon 


\section{INTRODUCTION}

Recent experimental data suggest the neurogenic origin of cardiac arrhythmias [5]. Remodelling of neuronal function and altered synaptic plasticity have been implicated in myocardial infarction and in wide spectrum of neurological diseases [10]. In order to understand the role of the intracardiac nervous system, many studies have investigated the morphology, neurochemistry and function of intracardiac neurons and intracardiac nerves of various animal models $[1-3,8]$. Nonetheless, synaptic connections of the intracardiac neurons remain poorly understood so far.

The frog is a convenient animal model to study the electrophysiology, development and structure of intracardiac neurons. The frog heart contains 11001600 intrinsic neurons [1]. Compared to the mammalian heart, frog intracardiac neurons are large and survive well in tissue preparations [6]. The frog heart receives parasympathetic innervation by the branches of the right and left vagus nerves that extend from the venal sinus into the interatrial septum and then into the ventricles [1]. Preganglionic fibres form synaptic boutons on the intracardiac neurons. During synaptic transmission, acetylcholine is released into the synaptic cleft from vesicles that are stored in synaptic boutons. Although the distribution of frog intracardiac neurons is well known [1], the size and distribution of synaptic boutons on frog intracardiac neurons remains to be elucidated.

In the present study, we investigate the innervation of intracardiac neurons by preganglionic axons in the frog Rana temporaria. We aimed to determine the quantitative distribution of synaptic boutons on frog intracardiac neurons.

\section{MATERIAL AND METHODS}

The study was performed on four adult (20-35 g in weight) common frogs (Rana temporaria). The investigation conforms to both the "Principles of laboratory animal care" (NIH publication No 86-23, revised 1985) and the local guidelines for the use of experimental animals. The frogs were euthanized by decapitation.

\section{Immunohistochemistry}

The atria with the interatrial septum, venal sinus and atrio-ventricular region were dissected and pinned flat in a Petri dish with physiologic solution. The preparation was double labelled immunohistochemically for the 
parasympathetic marker choline acetyltransferase (ChAT) and the marker of synaptic vesicles synaptophysin (SYP) as described previously [3]. We used goat anti-ChAT (AP144P, Chemicon, USA, 1:100) and rabbit anti-SYP (AB9272, Chemicon, USA, 1:500) primary antibodies as well as anti-goat Cy3-conjugated (AP180C, Chemicon, USA) and anti-rabbit FITC-conjugated (AP182F, Chemicon, USA) secondary antibodies. The preparations were kept at $4^{\circ}$ in primary anti-ChAT antibodies for 42 hours, and in primary anti-SYP antibodies for 14 hours.

\section{Confocal microscopy and measurements}

To photograph intracardiac neurons in situ at a high resolution, we used a confocal laser scanning microscope LSM 700/Axio Observer.Z1 (Zeiss, Germany). The labelled neurons were optically sectioned at $0.3 \mu \mathrm{m}$ by the aid of a $63 \times$ oil immersion objective and ZEN 2010 software (Zeiss, Germany). The extended focus method was used to reconstruct neuronal images from stacks of optical sections. One hundred labelled neurons were selected for analysis. The areas of neuronal soma and synaptic boutons were measured in extended focus images of neurons. Based on the neuronal soma area, we subdivided the neuronal soma into the axonal half and the non-axonal half. For comparison with previous studies, the 3D surface area of the neuron was calculated based on the short and the long axis of the neuron and the formula for the surface area of an oblate spheroid [8]. Comparison of measurements was performed with non-parametric Friedman test for related samples (SPSS 22.0). Significance was accepted at $\mathrm{P}<0.001$.

\section{RESULTS}

Synaptic boutons were found on both the axonal and the non-axonal half of the intracardiac neuron soma as well as on the proximal axon (Fig. 1). The total number of synaptic boutons was $10 \pm 2(\operatorname{mean} \pm S E ; \min =2 ; \max =121)$. In some cases counting of synaptic boutons was difficult due to their high density. The largest area of synaptic boutons was found on the axonal half of the soma ( $59 \pm 4$ $\left.\mu \mathrm{m}^{2} ; \min =0 \mu \mathrm{m}^{2} ; \max =239 \mu \mathrm{m}^{2}\right)$. The area of synaptic boutons on the nonaxonal half of the soma was smaller $\left(36 \pm 3 \mu \mathrm{m}^{2} ; \min =0 \mu \mathrm{m}^{2} ; \max =123 \mu \mathrm{m}^{2}\right)$. The smallest area of synaptic boutons was found on the proximal axon $(6 \pm 2$ $\left.\mu \mathrm{m}^{2} ; \min =0 \mu \mathrm{m}^{2} ; \max =72 \mu \mathrm{m}^{2}\right)$. The areas of synaptic boutons in all the three regions of the neuron differed significantly (Fig. 2 ; P $<0.001$ ). 
The total area of neuronal soma profile in extended focus projection image was $793 \pm 33 \mu \mathrm{m}^{2}\left(\min =255 \mu \mathrm{m}^{2} ; \max =1888 \mu \mathrm{m}^{2}\right)$. The total area of synaptic boutons per neuron was $98 \pm 6 \mu \mathrm{m}^{2}\left(\min =6 \mu \mathrm{m}^{2} ; \max =270 \mu \mathrm{m}^{2}\right)$. Synaptic boutons occupied $12.8 \pm 0.6 \%$ of the neural soma in extended focus projection image $(\min =1 \% ; \max =29 \%)$. Calculations of neuronal soma $3 \mathrm{D}$ area revealed that synaptic boutons occupied $2.8 \pm 0.1 \%(\min =0.3 \%$; $\max =7.6 \%)$ of the $3 \mathrm{D}$ area of the neuronal soma.
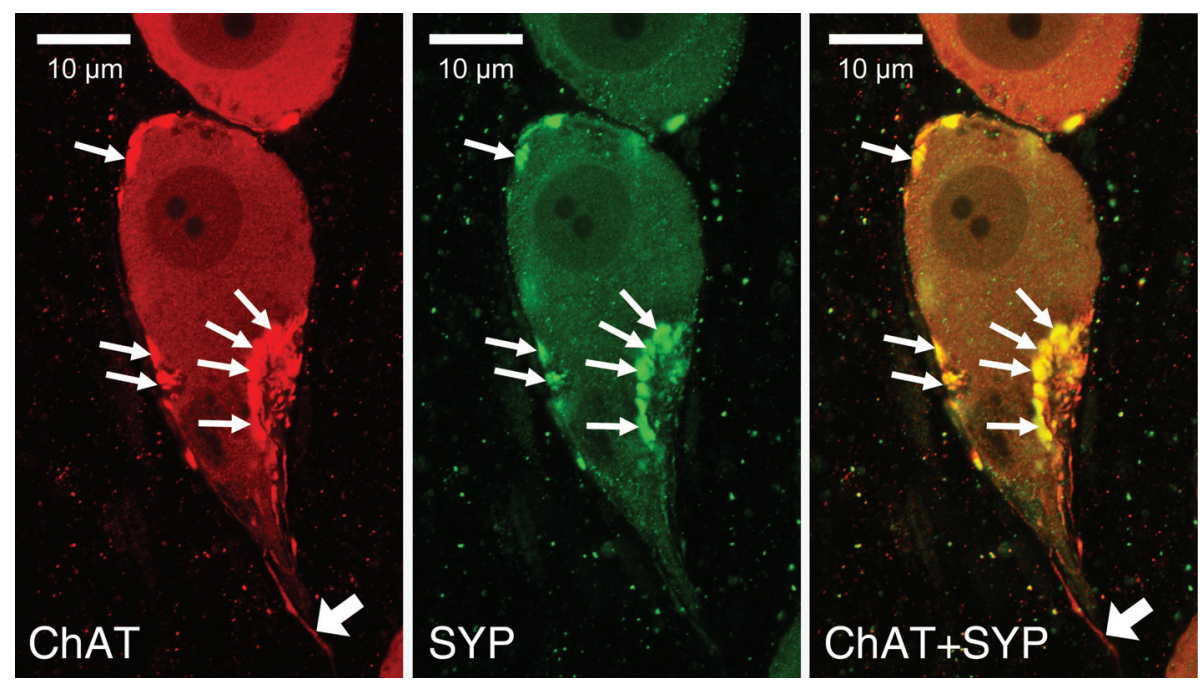

Figure 1. Frog intracardiac neuron double labelled for choline acetyltransferase (ChAT) and synaptophysin (SYP). Thin arrows point to some synaptic boutons that are clustered on both the axonal and the non-axonal half of the soma. The thick arrow demonstrates a terminal of preganglionic fibre that is positive for ChAT but negative for SYP.

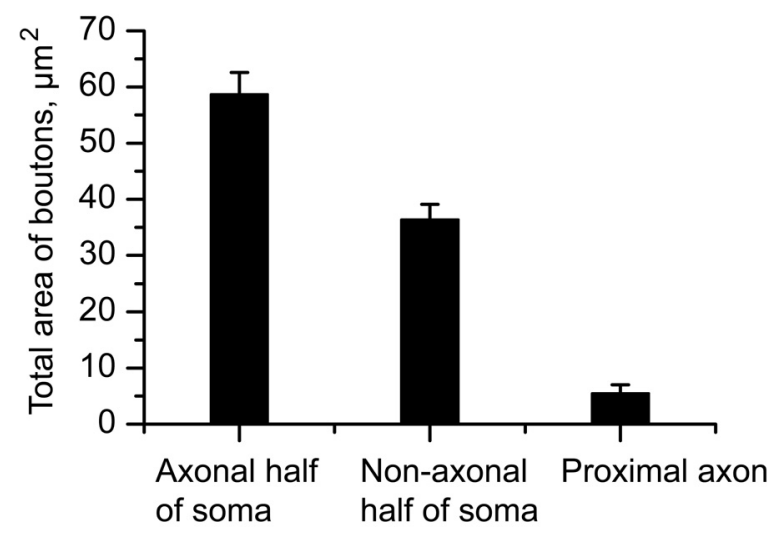

Figure 2. Total area of synaptic boutons (in $\mu \mathrm{m}^{2}$ ) on the axonal half of the soma, the non-axonal half of the soma and the proximal axon of frog intracardiac neurons $(n=100)$. All values are significantly different at $\mathrm{P}<0.001$. 


\section{DISCUSSION}

Our study demonstrates that frog intracardiac neurons exhibit great variability in the number and size of synaptic boutons. Distribution of synaptic boutons on the soma of the frog intracardiac neuron is uneven. Most of synaptic boutons cluster on the axonal pole of the neuronal soma, fewer boutons are distributed on the non-axonal half of the soma. Finally, few boutons are positioned on the proximal axon. Hence, we consider that axosomatic and axoaxonic synapses are formed by preganglionic fibre terminals on frog intracardiac neurons.

Our findings supplement earlier data on the anatomy of synaptic boutons of intracardiac neurons in amphibians by assessing quantitatively synaptic boutons in the heart of the frog Rana temporaria. Earlier it has been reported that in total $2 \%$ of the neuronal body is covered by synaptic boutons in the frog Xenopus laevis, about 3\% in the frog Rana pipiens and 5\% in the mudpuppy, Necturus maculosus $[6,7,9]$. The total mean area of synaptic boutons determined in our study is similar to other amphibian species $[6,7,9]$.

Although it was considered that frog intracardiac neurons have no dendrites [4], previous study has shown abundance of dendrite-like processes on the hillock and the proximal axon of frog intracardiac neurons [1]. These processes do not terminate with synaptic boutons [1]. Taking into account the high abundance of synaptic boutons on the hillock and the proximal axon, it is likely that these processes function as dendrites that facilitate neuronal contacts with the terminals of preganglionic fibres. Further combined immunohistochemical, intracellular filling and electron microscopy studies are needed to prove the synaptic contacts between the terminals of preganglionic fibres and dendrite-like processes on the hillock and the proximal axon of frog intracardiac neurons.

\section{CONCLUSIONS}

1) The axonal half of the soma of the frog intracardiac neuron is more densely innervated than the non-axonal half of the soma. 2) Axosomatic and axoaxonic synapses are present on frog intracardiac neurons. This study provides a framework for further experimental studies on formation and rearrangement of synapses on frog intracardiac neurons. 


\section{REFERENCES}

1. Batulevicius D., Skripkiene G., Batuleviciene V., Skripka V., Dabuzinskiene A., Pauza D.H. (2012). Distribution, structure and projections of the frog intracardiac neurons. Auton Neurosci, 168, 1-2, 14-24.

https://doi.org/10.1016/j.autneu.2012.01.004

2. Batulevicius D., Frese T., Peschke E., Pauza D.H., Batuleviciene V. (2013). Remodelling of the intracardiac ganglia in diabetic Goto-Kakizaki rats: an anatomical study. Cardiovasc Diabetol, 12, 85. https://doi.org/10.1186/1475-2840-12-85

3. Brack. K.E. (2015). The heart's 'little brain' controlling cardiac function in the rabbit. Exp Physiol, 100, 348-353.

https://doi.org/10.1113/expphysiol.2014.080168

4. Gibbins I.L., Morris J.L. (2006). Structure of peripheral synapses: autonomic ganglia. Cell Tissue Res, 326, 205-220.

https://doi.org/10.1007/s00441-006-0233-1

5. Kalla M., Herring N., Paterson D.J. (2016). Cardiac sympatho-vagal balance and ventricular arrhythmia. Auton Neurosci, 199, 29-37.

https://doi.org/10.1016/j.autneu.2016.08.016

6. McMahan U.J., Kuffler S.W. (1971). Visual identification of synaptic boutons on living ganglion cells and of varicosities in postganglionic axons in the heart of the frog. Proc R Soc Lond B Biol Sci, 177, 485-508.

https://doi.org/10.1098/rspb.1971.0044

7. McMahan U.J., Purves D. (1976). Visual identification of two kinds of nerve cells and their synaptic contacts in a living autonomic ganglion of the mudpuppy (Necturus maculosus). J Physiol, 405-425.

https://doi.org/10.1113/jphysiol.1976.sp011238

8. Rysevaite K., Saburkina I., Pauziene N., Vaitkevicius R., Noujaim S.F., Jalife J., Pauza D.H. (2011). Immunohistochemical characterization of the intrinsic cardiac neural plexus in whole-mount mouse heart preparations. Heart Rhythm, 8 , 731-738. https://doi.org/10.1016/j.hrthm.2011.01.013

9. Sargent P.B. (1983). The number of synaptic boutons terminating on Xenopus cardiac ganglion cells is directly correlated with cell size. J Physiol, 85-104. https://doi.org/10.1113/jphysiol.1983.sp014882

10. Zhang D., Tu H., Wang C., Cao L., Muelleman R.L., Wadman M.C., Li Y (2017). Correlation of ventricular arrhythmogenesis with neuronal remodeling of cardiac postganglionic parasympathetic neurons in the late stage of heart failure after myocardial infarction. Front Neurosci, 08 May.

https://doi.org/10.3389/fnins.2017.00252 
Address for correspondence:

Darius Batulevičius

Institute of Anatomy

Faculty of Medicine

Lithuanian University of Health Sciences

A. Mickevičiaus 9, LT-44307 Kaunas, Lithuania

E-mail: darius.batulevicius@lsmuni.lt; phone: +370 37327295 